\title{
Reversing effect and mechanism of soluble resistance-related calcium-binding protein on multidrug resistance in human lung cancer A549/DDP cells
}

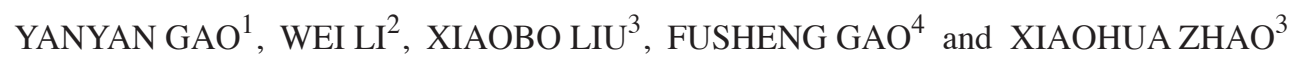 \\ ${ }^{1}$ Department of Internal Medicine, Weifang Medical University, Weifang, Shandong 261053; ${ }^{2}$ Department of Chest Surgery, \\ Weifang People's Hospital, Weifang, Shandong 261041; Departments of ${ }^{3}$ Chest Surgery and ${ }^{4}$ Respiratory Medicine, \\ The Affiliated Hospital of Weifang Medical University, Weifang, \\ Shandong 261041, P.R. China
}

Received November 2, 2013; Accepted April 7, 2014

DOI: $10.3892 / \mathrm{mmr} .2014 .2936$

\begin{abstract}
Lung cancer is the primary malignancy of the lung and is the leading cause of cancer-associated mortality in China. Multidrug resistance (MDR) is an essential aspect of lung cancer treatment failure and a popular topic of investigation in tumor studies. Previous studies have demonstrated that soluble resistance-related calcium-binding protein (Sorcin) is involved in the MDR of various types of human tumor, and that silencing Sorcin was able to reverse the MDR of several types of cultured human cancer cells. However, the effect and potential mechanism underlying the ability of Sorcin to reverse MDR in human lung cancer remains to be fully elucidated. The present study examined the role of Sorcin in the reversal of MDR in human lung cancer A549/DDP cells. The effects included increased drug sensitivity to cisplatin, apoptotic rate, cell cycle arrest in the $\mathrm{G} 2 / \mathrm{M}$ phase and intracellular accumulation of rhodamine-123, and decreased expression of multidrug resistance gene 1 , lung resistance protein, multidrug resistance-associated protein, glutathione S-transferase $\pi$, ATP-binding cassette transporter A2 (ABCA2), ABCA5, B-cell lymphoma 2 and P-glycoprotein, and the depletion of glutathione in Sorcin-silenced A549/DDP cells. The present study also revealed that there was a downregulation of $\mathrm{p}-\mathrm{Akt}$ and phosphorylated extracellular signal-regulated kinase (p-ERK), and a decreased transcriptional activation of nuclear factor $\kappa \mathrm{B}$, signal transducer and activator of transcription (STAT)3, STAT5 and nuclear factor of activated T-cells following silencing of Sorcin. The results indicated that Sorcin
\end{abstract}

Correspondence to: Mr. Xiaohua Zhao, Department of Chest Surgery, The Affiliated Hospital of Weifang Medical University, 2428 Yuhe Road, Kuiwen, Weifang, Shandong 261041, P.R. China E-mail: xiaohua_zhaosd@126.com

Key words: lung cancer, soluble resistance-related calcium-binding protein, multi-drug resistance may be used as a potential therapeutic target for MDR through inhibiting the Akt and ERK pathways in human lung cancer.

\section{Introduction}

Lung cancer is the most common tumor worldwide. Surgical resection to remove the tumor together with surrounding lung tissue remains the best treatment for this disease. However, only $10-15 \%$ of patients are suitable for surgical resection (1). Cisplatin is the major component of the chemotherapeutic combination that is used in transcatheter arterial chemoembolization (cisplatinum, interferon, doxorubicin and 5-fluorouracil). However, the drug resistance of lung cancer to chemotherapy remains a major challenge (2). There are several intrinsic or acquired mechanisms that have been elucidated. Lung cancer cells frequently possess intrinsic drug resistance mediated by enhanced cellular drug efflux of several cytotoxic agents. This phenomenon is associated with an increased expression of ATP-binding cassette (ABC) proteins, a drug transporter family, including multidrug resistance gene 1 (MDR1) and multidrug resistance-associated protein (MRP1) (3).

Soluble resistance-related calcium-binding protein (Sorcin) is a $22 \mathrm{kDa}$ calcium-binding protein that was initially found to be overexpressed in vincristine-resistant cells. The binding of $\mathrm{Ca}^{2+}$ triggers the translocation of Sorcin from the cytoplasm to the cell membrane, where it interacts with specific proteins involved in the signaling cascades of several physiological processes (4). Further studies revealed that Sorcin was not only overexpressed in vincristine-resistant cells, but also in several tumor cell types with different drug-resistant profiles (5). Previous studies indicated that overexpression of Sorcin is associated with resistance in human ovarian, breast cancer, lung cancer, leukemia and gastric carcinoma (6). Downregulation of Sorcin expression in K562/A02 cells, which are resistant to doxorubicin, restored drug sensitivity (7-9). These findings suggest that Sorcin may be a potential therapeutic target for reversing MDR in cancer. However, the role of Sorcin in lung cancer MDR remains to be elucidated. 
The current study aimed to investigate the effect of Sorcin on multidrug resistance reversal of human lung cancer A549/DDP cells and its mechanism.

\section{Materials and methods}

Cell lines and cell culture. The human lung cancer cell line A549 and cisplatin resistant cell line A549/DDP were purchased from the Cell Bank of the Chinese Academy of Sciences (Shanghai, China) and maintained at $37^{\circ} \mathrm{C}$ in a humidified atmosphere with $5 \% \mathrm{CO}_{2}$. The culture medium was Eagle's minimal essential medium supplemented with $10 \%$ fetal bovine serum (FBS; Gibco-BRL, Carlsbad, CA, USA). The present study was approved by the Ethics Committee of Weifang Medical University (Weifang, China).

Silencing of Sorcin by siRNA. The Sorcin siRNA and blank vector were purchased from Santa Cruz Biotechnology, Inc. (Santa Cruz, CA, USA) and the transfection of siRNA was performed according to the manufacturer's instructions. Briefly, $4 \times 10^{5}$ A549/DDP cells were seeded into 6-well plates for $24 \mathrm{~h}$ in the culture medium. Then, Sorcin siRNA and the blank vector were transfected with Lipofectamine 2000. Following $24 \mathrm{~h}$ of incubation, normal growth medium containing $15 \%$ FBS was added and the cells were incubated for another $18 \mathrm{~h}$. The medium was aspirated and replaced with fresh normal growth medium (10\% FBS) and was added after $72 \mathrm{~h}$ with $400 \mu \mathrm{g} / \mathrm{ml} \mathrm{G} 418$ to generate Sorcin silencing and negative control sublines. Sorcin expression was measured by western blotting and quantitative polymerase chain reaction (qPCR).

MTS assay to determine the drug sensitivity of the cells. The cells $(0.1 \mathrm{ml})$ were seeded into each well of the 96-well microplate at a density of $1 \times 10^{5} / \mathrm{ml}$ and incubated overnight to allow the adherence of cells. Then, different concentrations of cisplatin (Sigma-Aldrich, St. Louis, MO, USA) were added to each well and the cells were cultured for another $72 \mathrm{~h}$. At the end of the incubation, CellTiter 96 Aqueous One Solution Reagent (Promega Corporation, Madison, WI, USA) was employed according to the manufacturer's instructions. After $4 \mathrm{~h}$, the cell viability was determined by measuring the absorbance at $490 \mathrm{~nm}$ using a microplate reader (US Biotek Laboratories, Seattle, WA, USA).

Analysis of intracellular accumulation of Rhod-123, membrane expression of $P$-gp, the cell cycle and cell apoptosis using flow cytometry. Approximately $3 \times 10^{5}$ cells were seeded into each well of the 6-well microplate and incubated with $10 \mu \mathrm{M}$ cisplatin for $24 \mathrm{~h}$. Cells were then harvested and the apoptosis rate was determined using an Annexin V-FITC Apoptosis kit purchased from BD Biosciences (Franklin Lakes, NJ, USA). Briefly, $20 \mu 1$ of Annexin V-FITC and propidium iodide (PI) were added to the suspension and incubated for $15 \mathrm{~min}$ in the dark at room temperature. Subsequently, the cells were washed twice with phosphate-buffered saline (PBS), resuspended with PBS and analyzed by flow cytometry at an excitation wavelength of $488 \mathrm{~nm}$.

Approximately $1 \times 10^{6}$ cells were harvested and fixed in cold alcohol at $4^{\circ} \mathrm{C}$ overnight. The cells were washed twice with PBS, stained with $10 \mu \mathrm{M}$ PI with DnaseA for $30 \mathrm{~min}$ and analyzed using flow cytometry (FACSCalibur; BD Biosciences) at an excitation wavelength of $488 \mathrm{~nm}$.

Approximately $1 \times 10^{6}$ cells were harvested and resuspended in $0.1 \mathrm{ml}$ culture medium. The cells were stained with $10 \mu \mathrm{M}$ Rhod-123 (Sigma, St. Louis, MO, USA) for $1 \mathrm{~h}$ and the intracellular concentration was determined by flow cytometry (FACSCalibur; BD Biosciences) at an excitation wavelength of $488 \mathrm{~nm}$.

The expression of P-gp on the cell surface was determined using a direct immunofluorescence staining kit purchased from BD Biosciences. Briefly, $20 \mu \mathrm{l}$ of reagent containing anti-P-gp-PE was added to the suspension and incubated for $30 \mathrm{~min}$ in the dark at room temperature. Cells were then washed twice with PBS, resuspended with $0.5 \mathrm{ml}$ of $1 \%$ paraformaldehyde and analyzed by flow cytometry at an excitation wavelength of $488 \mathrm{~nm}$.

Determination of glutathione (GSH) expression levels. The intracellular GSH was measured using a Total Glutathione Assay kit (Beyotime Institute of Biotechnology, Haimen, Jiangsu, China). Analysis was performed according to the manufacturer's instructions. Briefly, cells were incubated with cisplatin for $4 \mathrm{~h}$. Cell lysates were harvested and reacted with assay solution for $5 \mathrm{~min}$ at $25^{\circ} \mathrm{C}$. The absorbance at $412 \mathrm{~nm}$ was measured on a Spectra Max M5 microplate reader (Molecular Devices, Sunnyvale, CA, USA).

$q P C R$ analysis. Approximately $3 \times 10^{6}$ cells were harvested for qPCR analysis. Total mRNA was extracted from the cells using a Dynabeads mRNA Direct kit (Invitrogen Life Technologies, Carlsbad, CA, USA) according to the manufacturer's instructions. Total mRNA was then reverse transcribed for $1 \mathrm{~h}$ at $42^{\circ} \mathrm{C}$ in incubation buffer containing $250 \mu \mathrm{M}$ of each deoxynucleotide triphosphate, $5 \mu \mathrm{M}$ oligo (dT)20, 25 units of RNase inhibitor and 20 units of avian myeloblastosis virus reverse transcriptase (Roche Diagnostics, Mannheim, Germany). The transcription level of MDR1, MRP1, ABCA2, ABCA5 and Bcl-2 was detected by semiquantitative PCR using the iCycler iQ detection system (Bio-Rad, Hercules, CA, USA). The PCR conditions were as follows: decontamination at $65^{\circ} \mathrm{C}$ for 2 min, denaturation at $95^{\circ} \mathrm{C}$ for $2 \mathrm{~min}$, followed by 40 cycles at $95^{\circ} \mathrm{C}$ for $20 \mathrm{sec}$ and at $65^{\circ} \mathrm{C}$ for $40 \mathrm{sec} . \beta$-actin was used as the internal control. The full details are shown in Table I.

Western blotting. Cells were eliminated by trypsinization and the whole proteins were obtained using radioimmunoprecipitation assay lysis buffer (Millipore, Billerica, MA, USA) extraction and centrifugation at $12,000 \mathrm{x}$ g for $10 \mathrm{~min}$. Total protein concentrations of the supernatants were measured using the bicinchoninic acid kit (Sigma-Aldrich, St. Louis, MO, USA). Proteins $(20 \mu \mathrm{g})$ were separated on $12 \%$ sodium dodecyl sulfate polyacrylamide gel electrophoresis and transferred onto polyvinylidene fluoride membranes. The membranes were blocked for $2 \mathrm{~h}$ at room temperature in Tris-NaCl-Tween (TNT) buffer (10 mM Tris- $\mathrm{HCl}$ and $150 \mathrm{mM} \mathrm{NaCl}, \mathrm{pH} 7.4,0.1 \%$ Tween-20) with 5\% non-fat dried milk, followed by incubation overnight at $4^{\circ} \mathrm{C}$ with rabbit anti-MDR1, MRP1, LRP, GST- $\pi$, ABCA2, ABCA5, Bcl-2, p-ERK, p-Akt and $\beta$-actin antibody 
Table I. Oligonucleotide sequences for quantitative polymerase chain reaction.

Gene Sequence (5'-3')

MDR1 Forward: AAAAAGATCAACTCGTACCACTC Reverse: GCACAAAATACACCAACAA

MRP1 Forward: ACTTCCACATCTGCTTCGTCAGTG Reverse: ATTCAGCCACAGGAGGTAGAGAGC

LRP Forward: GCCTGACTTCTTCACAGACGTC Reverse: TCA AAGTGCCAGTTGTAGGCC

GST- $\pi$ Forward: CTGGAAGGAGGAGGTGGTG Reverse: GACGCAGGATGGTATTGGAC

ABCA2 Forward: CCCGGAAGATTGGCCGTATCCTGG Reverse: TTGAAGGACAGCTGGGCCCGC

ABCA5 Forward:GATGATTCACTGAAGTGTATGGGTTA Reverse: ATCTTAACTGCCCAGACACCATGAT

BCL-2 Forward: ACGGGGTGAACTGGGGGAGGA Reverse: TGTTTGGGGCAGGCATGTTGACTT

$\beta$-actin Forward: TGAGCGCGGCTACAGCTT Reverse: TCCTTAATGTCACGCACGATTT

MDR1, multidrug resistance gene 1; MRP1, multidrug resistance-associated protein 1; LRP, lung resistance protein; GST- $\pi$, glutathione S-transferase $\pi$; ABC, ATP-binding cassette transporter; Bcl-2, B-cell lymphoma 2.

(Santa Cruz Biotechnology, Inc.). All primary antibodies were diluted according to the manufacturer's instructions. The membranes were washed and incubated for $1 \mathrm{~h}$ with peroxidase-labelled anti-rabbit IgG (Santa Cruz Biotechnology, Inc., diluted at 1:2,000). Finally, the membranes were washed three times in TNT buffer and exposed to the Immobilon $^{\mathrm{TM}}$ western chemiluminescent horseradish peroxidase substrate (Millipore) for $1 \mathrm{~min}$, and then exposed to autoradiography film for 2-3 min in the dark. $\beta$-actin was used as the internal control.

NF- $\kappa B$, STAT3, STAT5 and NFAT transcriptional activities assay. The activity of NF- $\mathrm{KB}$ was determined by the reporter gene system (Promega Corporation) according to the manufacturer's instructions with moderate modification. Briefly, $1 \times 10^{4}$ cells were seeded into each well of a 96-well plate and incubated overnight to allow the adherence of cells. Then, the activity of transcription factors was measured using a TransAM transcription factor activity ELISA kit (Active Motif, Carlsbad, CA, USA) according to the manufacturer's instructions.

Statistical analysis. Data are expressed as the mean \pm standard deviation. Statistical analysis was performed using one-way analysis of variance and $\mathrm{P}<0.05$ was considered to indicate a statistically significant difference.

\section{Results}

Sorcin siRNA transfection results in downregulation of Sorcin expression. As shown in Fig. 1, the Sorcin level in
A549/DDP cells was elevated significantly compared with the parental A549 cells. After A549/DDP cells were transfected with Sorcin siRNA, the Sorcin level in A549/DDP Sorcin silenced cells was decreased significantly compared with the normal A549/DDP cells. With different silencing levels of Sorcin, A549/DDP cells were divided into the Sorcin siRNA group 1 and Sorcin siRNA group 2. A549/DDP cells transfected with control siRNA were the negative group, A549/DDP cells without treatment were the control group and A549 cells were the parent group.

Sorcin silencing increases cisplatin sensitivity. The present study determined the sensitivity of the cells to cisplatin following Sorcin silencing using an MTS assay to examine whether Sorcin contributed to the cisplatin resistance of A549/DDP. The drug sensitivity of each group of cells was represented by the $\mathrm{IC}_{50}$. As shown in Fig. 2, the $\mathrm{IC}_{50}$ values of the Sorcin silenced cells were lower than that of the parental A549/DDP cells. The $\mathrm{IC}_{50}$ of the Sorcin siRNA group 1 and the Sorcin siRNA group 2 were 36.4 and $17.6 \mu \mathrm{M}$, respectively, however, the $\mathrm{IC}_{50}$ of the control group and the negative group were 46.8 and $45.6 \mu \mathrm{M}$, respectively, suggesting that the reversal fold of Sorcin silencing were 1.29 and 2.66 fold, respectively [Reversal fold $=\mathrm{IC}_{50}$ (resistance cells) $/ \mathrm{IC}_{50}$ (reversal cells)]. These results indicated that Sorcin has a positive function in the cisplatin resistance of the cells.

Sorcin silencing increases cell apoptosis, cell cycle arrest in the G2/M phase and intracellular accumulation of Rhod-123, and decreases the expression of $P$-gp. It was possible that the restoration of cisplatin sensitivity by Sorcin silencing was associated with the regulation of accumulation of intracellular drug, since increasing drug efflux is a major drug resistance mechanism. Rhod-123 is a fluorescent pigment that shares the same membrane transporter protein with cisplatin and is able to reflect the intracellular drug accumulation potency. The results from the flow cytometry demonstrated that the Sorcin silenced cells have a greater intracellular fluorescent activity, which indicated that more Rhod-123 was retained in the cell. In addition, flow cytometric analysis demonstrated that Sorcin silencing was able to downregulate the expression of P-gp, increase cell apoptosis and arrest the cell cycle in $\mathrm{G} 2 / \mathrm{M}$ phase, indicating that Sorcin silencing was able to restore cisplatin sensitivity through regulating the drug pump and the cell cycle in A549/DDP cells (Fig. 3).

Sorcin reverses $M D R$ by downregulating the expression of MDR1, MRP1, LRP, GST-л, ABCA2, ABCA5 and Bcl-2. MDR1, LRP and MRP1 are major membrane transporter proteins that lead to MDR due to their efflux activities. The present study was interested in whether transporters from other sub families of $\mathrm{ABC}$, including $\mathrm{ABCA} 2$ and $\mathrm{ABCA} 5$ were also involved in the drug resistance. GST- $\pi$ is a major antioxidant molecule leading to MDR in tumor cells. Bcl-2 has been implicated in several types of cancer and is also considered to be involved in resistance to conventional cancer treatment. Western blot analysis demonstrated that A549/DDP cells expressed a high level of MDR1, MRP1, LRP, GST- $\pi$, ABCA2, ABCA5 and Bcl-2. It was then investigated whether Sorcin was able to modulate the expression 
A

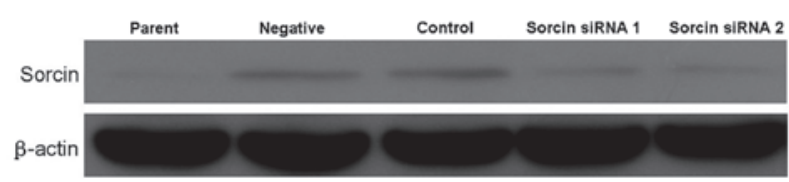

B

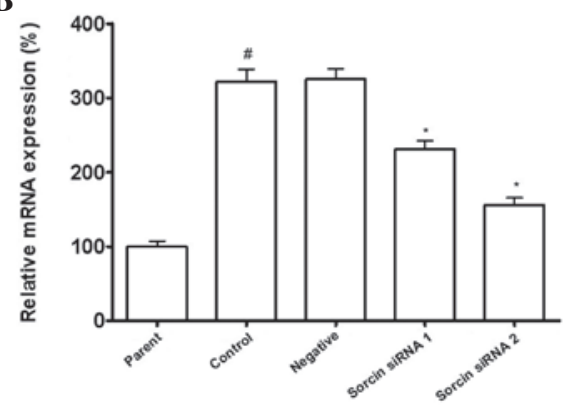

Figure 1. Expression level of Sorcin measured by western blot analysis and quantitative polymerase chain reaction following Sorcin silencing. (A) Protein expression of Sorcin with $\beta$-actin as a loading control $(n=3$ per lane). (B) mRNA expression of Sorcin with $\beta$-actin as a loading control. Data are expressed as the mean \pm standard deviation. ${ }^{\#} \mathrm{P}<0.05$, compared with the parent group; ${ }^{*} \mathrm{P}<0.05$, compared with the control group $(\mathrm{n}=5)$. Sorcin, soluble resistance-related calcium-binding protein; siRNA, small interfering RNA.

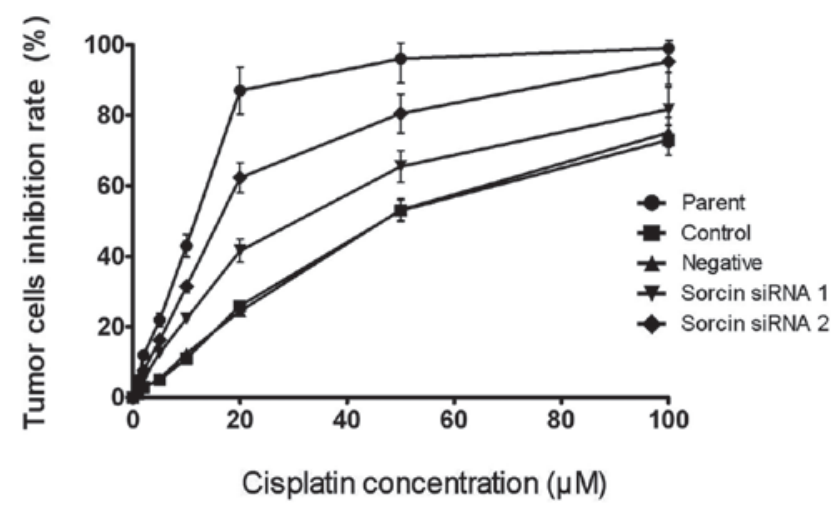

Figure 2. Effect of Sorcin silencing on cisplatin sensitivity measured by the MTS assay. Data are expressed as the mean \pm standard deviation, $n=10$. Sorcin, soluble resistance-related calcium-binding protein; siRNA, small interfering RNA.

of these proteins. The qPCR and western blotting results demonstrated that, when compared with the high expression level observed in the A549/DDP cells, the expression of MDR1, MRP1, LRP, GST- $\pi$, ABCA2, ABCA5 and Bcl-2 decreased in the Sorcin silenced cells (Fig. 4).

Intracellular level of GSH decreases as the cells gain sensitivity to cisplatin. Highly cisplatin resistant cancer cells are frequently associated with a marked increase of $\mathrm{GSH}$ synthesis. The reduced intracellular GSH was able to rescue the cells from cytotoxicity. The results demonstrated that the intracellular concentration of GSH was decreased in Sorcin-silenced A549/DDP cells compared with the control and negative groups. The intracellular concentration of GSH of the Sorcin siRNA group 1 and Sorcin siRNA group 2 were 52.7 and $31.6 \%$ compared with the control group, respectively (Fig. 5).
Sorcin modulates the phosphorylation level of AKT and ERK, and the transcriptional activities of $N F-\kappa B$, STAT3, STAT5 and NFAT. The present study aimed to investigate whether the PI3K/AKT and MEK/ERK pathways acted as linkers between Sorcin and drug resistance. The western blotting assays demonstrated that the phosphorylation level of AKT and ERK was downregulated in Sorcin-silenced A549/DDP cells (Fig. 6A).

$\mathrm{NF}-\kappa \mathrm{B}$ is activated by $\mathrm{PI} 3 \mathrm{~K} / \mathrm{AKT}$ and its activation can lead to the expression of drug resistance-associated genes, including MDR1 and MRP1. STAT3, STAT5 and NFAT are also shown to control EMT and the transition is reported to contribute to the chemoresistance of cancer cells. The present study aimed to elucidate whether NF- $\kappa$ B, STAT3, STAT5 and NFAT transcriptional activities were also modified by Sorcin. The reporter gene system assay demonstrated that the transcriptional activities of NF- $\kappa$ B, STAT3, STAT5 and NFAT were significantly decreased following Sorcin silencing (Fig. 6B).

\section{Discussion}

A large amount of evidence has connected the overexpression of Sorcin with MDR in cancer cells. However, the exact roles of Sorcin in cisplatin-resistant lung cancer remain to be elucidated. The present study demonstrated that in a human lung cancer cell line A549/DDP, which was resistant to cisplatin, the Sorcin protein was overexpressed. Furthermore, it was demonstrated that several mechanisms including drug efflux were involved in Sorcin-mediated drug resistance.

Lung cancer is one of the most common types of tumor worldwide. The treatment of lung cancer is of great important in China since approximately half of the patients worldwide are from China (10). Cisplatin is widely used as part of the combination chemotherapy (cisplatinum, interferon, doxorubicin and 5-fluorouracil) for unresectable disease. However, the response rate to this chemotherapy treatment is poor. Therefore, the investigation of the mechanisms involved in MDR is valuable for identifying new strategies to overcome the treatment failure. The present study aimed to elucidate whether Sorcin silencing was able to restore the drug sensitivity of A549/DDP. The MTS assay indicated an increased cisplatin cytotoxicity of A549/DDP cells by sorcin silencing. In other words, the sensitivity of cancer cells to cisplatin was inversely correlated with the cellular Sorcin level. This result was consistent with previous studies demonstrating that Sorcin contributed to the drug resistance of tumor cells.

One major mechanism involved in drug resistance is increasing the drug efflux from cancer cells $(11,12)$. The intracellular concentration of Rhod-123 and expression of P-gp were determined by flow cytometry. The results demonstrated that Sorcin silencing increased the intracellular concentration of Rhod-123 and decreased the expression of P-gp to reverse the drug resistance of tumor cells. MDR1, LRP and MRP1 are the most common membrane transporters that efflux drugs out of cells and are frequently found to be overexpressed in the drug resistant environment (13-15). The expression of these proteins in A549/DDP cells prior to and following Sorcin silencing was investigated. There was a significant downregulation of MDR1, LRP and MRP1 
A
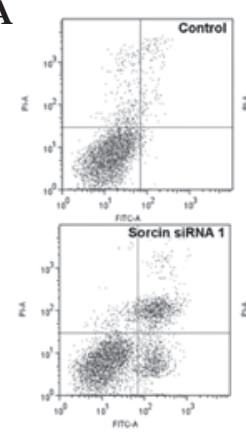

B

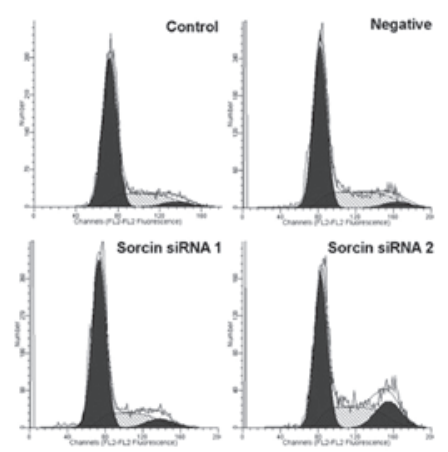

C
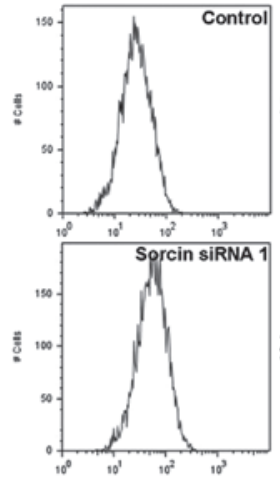

D

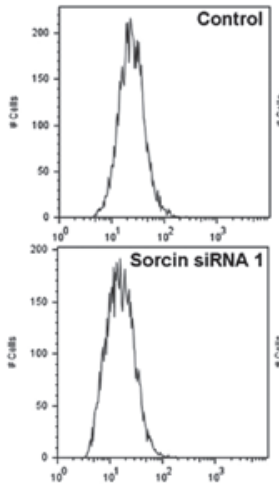

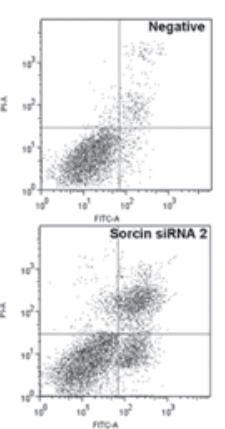
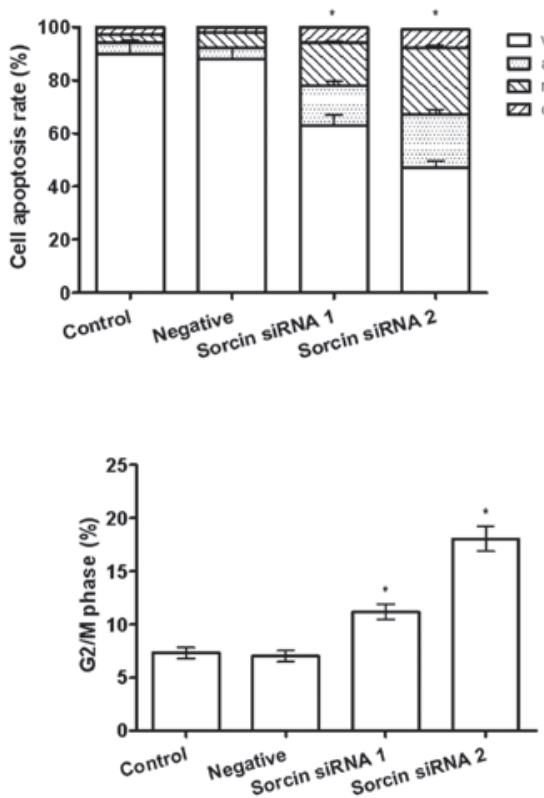

믈 viable cell
apoptosis cell
necrosis cell

西 dead cell
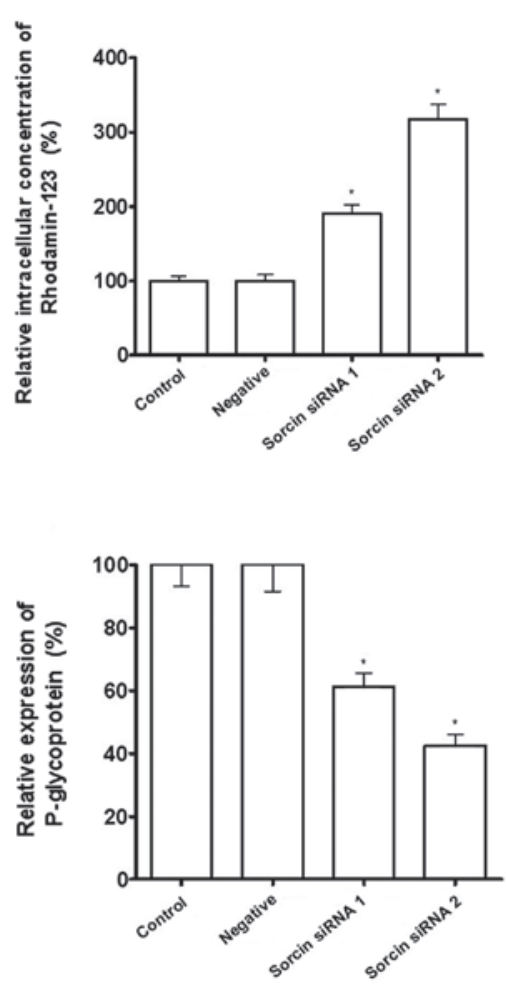

Figure 3. Effect of Sorcin silencing on drug efflux pumps measured by flow cytometry. (A) Percentage of apoptotic cells assessed by Annexin V and propidium iodide staining. " $\mathrm{P}<0.05$, compared with the control group, $\mathrm{n}=3$. (B) Percentage of cells arrested in the $\mathrm{G} 2 / \mathrm{M}$ phase of the cell cycle. ${ }^{*} \mathrm{P}<0.05$, compared with the control group, $\mathrm{P}<0.05, \mathrm{n}=3$. (C) Intracellular accumulation of drug assessed by rhodamine-123 staining. ${ }^{*} \mathrm{P}<0.05$, compared with the control group, $\mathrm{n}=3$. (D) Drug excretion assessed by P-glycoprotein staining. ${ }^{*} \mathrm{P}<0.05$, compared with the control group, $\mathrm{n}=3$. All the data are expressed as the mean \pm standard deviation. Sorcin, soluble resistance-related calcium-binding protein; siRNA, small interfering RNA.

mRNAs and proteins in Sorcin silenced A549/DDP cells, providing solid evidence to support the theory that these membrane transporters are involved in Sorcin-mediated cisplatin resistance of A549/DDP cells. It has been demonstrated that Sorcin possibly interacts with MDR1 through two distinct mechanisms (16), one is that the Sorcin and $M D R 1$ genes are located in the same homogeneously staining region, and they are most likely to co-amplify under drug selection pressure; the other is that MDR1 activation requires $\mathrm{Ca}^{2+}$, Sorcin can bind to $\mathrm{Ca}^{2+}$ with high affinity and regulate the concentration of $\mathrm{Ca}^{2+}$ and thus MDR1 activation. The mechanisms of MRP1 regulation remain to be elucidated, which makes our interpretation of the association between Sorcin and MRP1 difficult. 
A

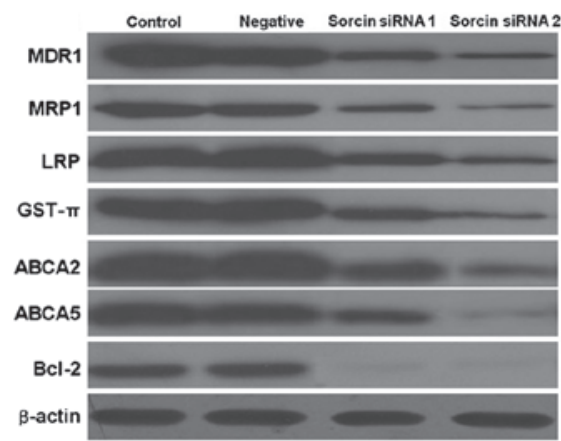

B

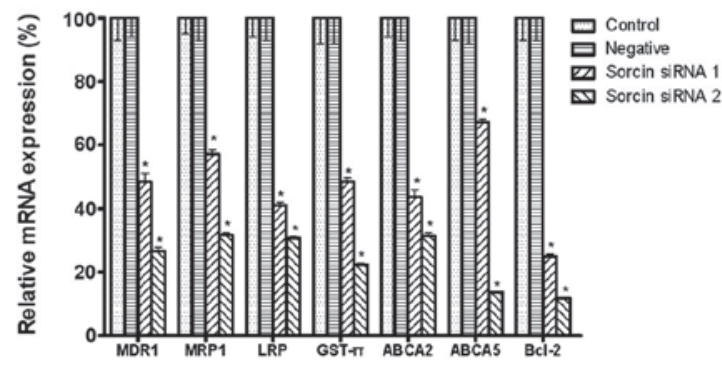

Figure 4. Expression level of multidrug resistance genes measured by western blot analysis and quantitative polymerase chain reaction following Sorcin silencing. (A) Protein expression of MDR1, MRP1, LRP, GST- $\pi$, ABCA2, ABCA5 and Bcl-2 with $\beta$-actin as a loading control (n=3 per lane). (B) mRNA expression of MDR1, MRP1, LRP, GST- $\pi$, ABCA2, ABCA5 and Bcl-2 with $\beta$-actin as a loading control. Data are expressed as the mean \pm standard deviation ${ }^{*} \mathrm{P}<0.05$, compared with the control group, $\mathrm{n}=5$. MDR1, multidrug resistance gene 1 ; MRP1, multidrug resistance-associated protein 1; LRP, lung resistance protein; GST- $\pi$, glutathione S-transferase $\pi$; ABC, ATP-binding cassette transporter; Bcl-2, B-cell lymphoma 2; Sorcin, soluble resistance-related calcium-binding protein; siRNA, small interfering RNA.

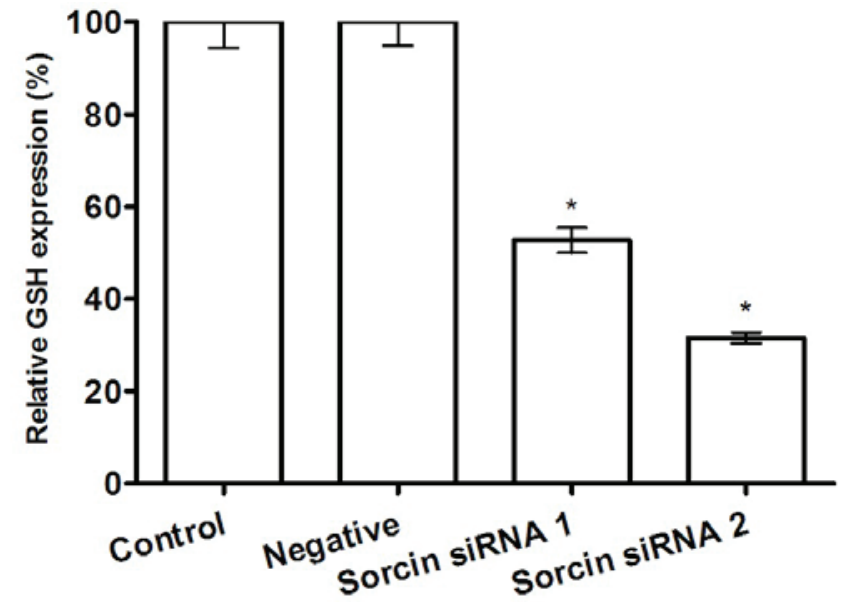

Figure 5. Effect of Sorcin silencing on the expression of GSH measured by ELISA. Data are expressed as the mean \pm standard deviation. ${ }^{*} \mathrm{P}<0.05$, compared with the control group $(\mathrm{n}=5)$. GSH, glutathione; siRNA, small interfering RNA.

MDR1 and MRP1 belong to the sub-family B and C of the $A B C$ family, respectively. Other sub families of $A B C$ are also involved in drug resistance since they pump the drugs out of the cells. The present study focused on ABCA2 and ABCA5, which are from the sub-family A. It has been reported that the overexpression of $\mathrm{ABCA} 2$ is a response to the resistance to estramustine (17). However, to the best of our knowledge, there are no studies investigating the role of ABCA2 and ABCA5 in cisplatin resistance. A considerable body of evidence highlights the importance of $\mathrm{ABC}$ proteins in cancer extending beyond drug transport to fundamental roles in tumor biology (18). Huang et al demonstrated that ABCA5 was upregulated in the stem cell-like side population of lung cancer PLC/PRF/5 cells. Drug resistance is one major property of cancer stem cells (19-21). The present study demonstrated that the expression of ABCA2 and 5 was elevated in A549/DDP cells and mediated by Sorcin. Whether cisplatin is the substrate of these two $\mathrm{ABC}$ proteins is unclear. There is a possibility that $\mathrm{ABCA} 2$ and 5 are important in cisplatin resistance in A549/DDP cells other than drug efflux, which requires further investigation.
A

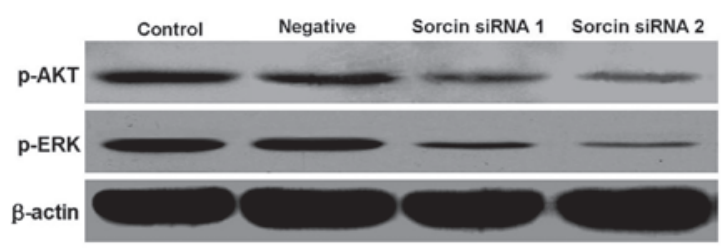

B

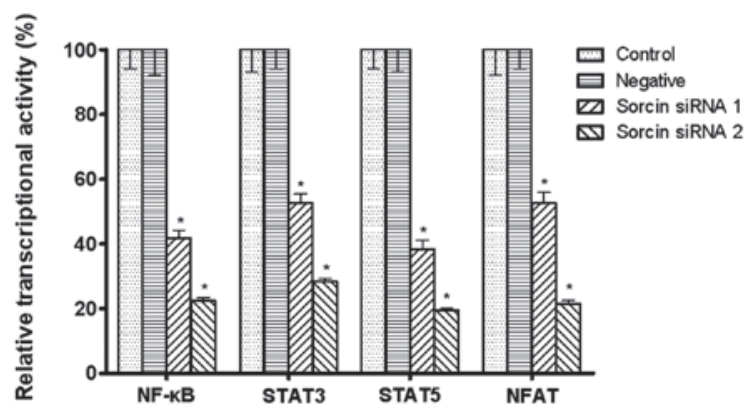

Figure 6. Effect of Sorcin silencing on signaling molecules measured by western blot analysis and enzyme-linked immunosorbent assay. (A) Phosphorylation of AKT and ERK with $\beta$-actin as a loading control $(n=3$ per lane). (B) Transcriptional activities of NF- $\mathrm{B}$, STAT3, STAT5 and NFAT. Data are expressed as the mean \pm standard deviation. ${ }^{*} \mathrm{P}<0.05$, compared with the control group, $n=5$. ERK, extracellular signal-regulated kinase; NF- $\kappa \mathrm{B}$, nuclear factor $\kappa \mathrm{B}$; STAT, signal transducer and activator of transcription; NFAT, nuclear factor of activated T-cells; Sorcin, soluble resistance-related calcium-binding protein; siRNA, small interfering RNA.

$\mathrm{Bcl}-2$ is a protein that has an anti-apoptotic effect in cancer cells. The finding that Bcl-2 was upregulated in A549/DDP cells indicated that the resistance to cisplatin-induced apoptosis is also involved in these cells. The regulation of Bcl-2 is complicated. The present study demonstrated that ERK activity was modified by Sorcin, which lead to the consequent regulation of $\mathrm{Bcl}-2$. In addition, it has also been reported that GSH is able to promote Bcl-2-mediated cisplatin resistance (22).

Other mechanisms are also involved in tumor resistance to cisplatin. One is GSH accumulation in cancer cells. The present study demonstrated that the level of GSH and GST- $\pi$ was downregulated following Sorcin silencing. The synthesis of GSH is mediated by several enzymes. $\gamma$-glutamylcysteine 
synthetase $(\gamma$-GCS), the rate-limiting enzyme for GSH biosynthesis, is found to be elevated in numerous cases of cisplatin resistance (23). Transcriptional and post-transcriptional regulations have been reported for the upregulation of $\gamma$-GCS (22).

Oxidative stress is able to stabilize the mRNA of $\gamma$-GCS through the MEK/ERK pathway (24). In the present study, the p-ERK level, which indicated the activity of MEK/ERK, was attenuated by Sorcin silencing. This may be the possible regulatory mechanism of $\gamma$-GCS in A549/DDP cells. It was revealed that Sorcin silencing led to the downregulation of AKT phosphorylation indicating that the PI3K/AKT pathway was modulated by Sorcin. Further investigation confirmed that the activity of NF- $\mathrm{KB}$, which is downstream to PI3K/AKT, was also inhibited by Sorcin silencing (25). To the best of our knowledge, there are no studies investigating the association between Sorcin and STAT3, STAT5 and NFAT. The present study was interested in these proteins since they are known to be involved in EMT, which is correlated with cancer malignancy and drug resistance (26-28).

In conclusion, by downregulating the expression of Sorcin in A549/DDP cells, the present study revealed that Sorcin was important in the drug resistance of A549/DDP cells. Targeting Sorcin remains a promising strategy to reverse drug resistance by several mechanisms. In addition, the PI3K/Akt and MEK/ERK pathways are important.

\section{References}

1. Sereno M, Rodríguez-Esteban I, Gómez-Raposo C, et al: Lung cancer and peritoneal carcinomatosis. Oncol Lett 6: 705-708, 2013

2. Chung AS, Wu X, Zhuang G, et al: An interleukin-17-mediated paracrine network promotes tumor resistance to anti-angiogenic therapy. Nat Med 19: 1114-1123, 2013.

3. Sadava D and Kane SE: Silibinin reverses drug resistance in human small-cell lung carcinoma cells. Cancer Lett 339: 102-106, 2013.

4. Li X, Wang J, Liu J, et al: Engagement of soluble resistance-related calcium binding protein (sorcin) with foot-and-mouth disease virus (FMDV) VP1 inhibits type I interferon response in cells. Vet Microbiol 166: 35-46, 2013.

5. $\mathrm{Hu} \mathrm{Y}$, Li S, Yang $\mathrm{M}$, et al: Sorcin silencing inhibits epithelial-to-mesenchymal transition and suppresses breast cancer metastasis in vivo. Breast Cancer Res Treat 143: 287-299, 2014.

6. Li GY, Liu JZ, Zhang B, et al: Tegillarca granosa extract Haishengsu (HSS) suppresses expression of mdr1, BCR/ABL and sorcin in drug-resistant K562/ADM tumors in mice. Adv Med Sci 58: 112-117, 2013.

7. Maddalena F, Laudiero G, Piscazzi A, et al: Sorcin induces a drug-resistant phenotype in human colorectal cancer by modulating $\mathrm{Ca}^{\left({ }^{+}\right)}$homeostasis. Cancer Res 71: 7659-7669, 2011.

8. Landriscina M, Laudiero G, Maddalena F, et al: Mitochondrial chaperone Trap1 and the calcium binding protein Sorcin interact and protect cells against apoptosis induced by antiblastic agents. Cancer Res 70: 6577-6586, 2010.

9. Hu Y, Cheng X, Li S, et al: Inhibition of sorcin reverses multidrug resistance of K562/A02 cells and MCF-7/A02 cells via regulating apoptosis-related proteins. Cancer Chemother Pharmacol 72 789-798, 2013.
10. Qu Y, Yang Y, Liu B, et al: Comparative proteomic profiling identified sorcin being associated with gemcitabine resistance in non-small cell lung cancer. Med Oncol 27: 1303-1308, 2010.

11. Kawakami M, Nakamura T, Okamura N, et al: Knock-down of sorcin induces up-regulation of MDR1 in HeLa cells. Biol Pharm Bull 30: 1065-1073, 2007.

12. Akazawa Y, Kawaguchi H, Funahashi M, et al: Effect of interferons on P-glycoprotein-mediated rhodamine-123 efflux in cultured rat hepatocytes. J Pharm Sci 91: 2110-2115, 2002.

13. Toner AP, McLaughlin F, Giles FJ, et al: The novel toluidine sulphonamide EL102 shows pre-clinical in vitro and in vivo activity against prostate cancer and circumvents MDR1 resistance. Br J Cancer 109: 2131-2141, 2013.

14. Tajitsu Y, Ikeda R, Nishizawa Y, et al: Molecular basis for the expression of major vault protein induced by hyperosmotic stress in SW620 human colon cancer cells. Int J Mol Med 32: 703-708, 2013.

15. Keppler D: Multidrug resistance proteins (MRPs, ABCCs): importance for pathophysiology and drug therapy. Handb Exp Pharmacol 201: 299-323, 2011.

16. Zheng BB, Zhang P, Jia WW, Yu LG and Guo XL: Sorcin, a potential therapeutic target for reversing multidrug resistance in cancer. J Physiol Biochem 68: 281-287, 2012.

17. Boonstra R, Timmer-Bosscha $\mathrm{H}$, van Echten-Arends $\mathrm{J}$, et al: Mitoxantrone resistance in a small cell lung cancer cell line is associated with $\mathrm{ABCA} 2$ upregulation. $\mathrm{Br} \mathrm{J}$ Cancer 90: 2411-2417, 2004.

18. Noguchi K, Katayama K and Sugimoto Y: Human ABC transporter ABCG2/BCRP expression in chemoresistance: basic and clinical perspectives for molecular cancer therapeutics. Pharmgenomics Pers Med 7: 53-64, 2014.

19. Huang L, Lu Q, Han Y, Li Z, Zhang Z and Li X: ABCG2/V-ATPase was associated with the drug resistance and tumor metastasis of esophageal squamous cancer cells. Diagn Pathol 7: 180, 2012.

20. Cuestas ML, Sosnik A and Mathet VL: Poloxamines display a multiple inhibitory activity of ATP-binding cassette (ABC) transporters in cancer cell lines. Mol Pharm 8: 1152-1164, 2011.

21. Visvader JE and Lindeman GJ: Cancer stem cells in solid tumours: accumulating evidence and unresolved questions. Nat Rev Cancer 8: 755-768, 2008.

22. Liu Y, Liu JH, Chai K, Tashiro S, Onodera S and Ikejima T: Inhibition of c-Met promoted apoptosis, autophagy and loss of the mitochondrial transmembrane potential in oridonin-induced A549 lung cancer cells. J Pharm Pharmacol 65: 1622-1642, 2013.

23. Kohsaka S, Takahashi K, Wang L, et al: Inhibition of GSH synthesis potentiates temozolomide-induced bystander effect in glioblastoma. Cancer Lett 331: 68-75, 2013.

24. Tang SC, Wu CH, Lai CH, et al: Glutathione S-transferase mu2 suppresses cancer cell metastasis in non-small cell lung cancer. Mol Cancer Res 11: 518-529, 2013.

25. Paolo M, Assunta S, Antonio R, et al: Selumetinib in advanced non small cell lung cancer (NSCLC) harbouring KRAS mutation: endless clinical challenge to KRAS-mutant NSCLC. Rev Recent Clin Trials 8: 93-100, 2013.

26. Dauphin M, Barbe C, Lemaire S, et al: Vimentin expression predicts the occurrence of metastases in non small cell lung carcinomas. Lung Cancer 81: 117-122, 2013.

27. Zhao G, Zhang JG, Shi Y, et al: MiR-130b is a prognostic marker and inhibits cell proliferation and invasion in pancreatic cancer through targeting STAT3. PLoS One 8: e73803, 2013.

28. Jans R, Mottram L, Johnson DL, et al: Lysophosphatidic acid promotes cell migration through STIM1- and Orail-mediated $\mathrm{Ca}^{2+}(\mathrm{i})$ mobilization and NFAT2 activation. J Invest Dermatol 133: 793-802, 2013. 